\title{
Simulation of RF-fields in a fusion device
}

\author{
Dieter De Witte*, Ignace Bogaert*, Daniel De Zutter*, Guido Van Oost ${ }^{\dagger}$ \\ and Dirk Van Eester** \\ ${ }^{*}$ Department of Information Technology (INTEC), Ghent University, Belgium \\ ${ }^{\dagger}$ Department of Applied Physics, Ghent University, Belgium \\ ${ }^{* *}$ Royal Military Academy, Brussels, Belgium
}

\begin{abstract}
In this paper the problem of scattering off a fusion plasma is approached from the point of view of integral equations. Using the volume equivalence principle an integral equation is derived which describes the electromagnetic fields in a plasma. The equation is discretized with MoM using conforming basis functions. This reduces the problem to solving a dense matrix equation. This can be done iteratively. Each iteration can be sped up using FFTs.
\end{abstract}

Keywords: RF heating, Method of Moments, Volume Integral Equation, Fast Fourier Transform PACS: 28.52.-S

\section{INTRODUCTION}

The simulation of radio frequency heating of a fusion plasma is a challenging computational task. Fortunately, the problem can be split up in different interacting subcomponents because different time scales can be identified in the plasma. One of those components for numerical simulation of the electromagnetic waves is the wave equation solver. A hot plasma can be described as a non-local anisotropic inhomogeneous medium. As a first step in the effort of tackling the problem the cold plasma approximation is used. The cold plasma approximation may seem a very rough approximation but it allows for a broad variety of wave phenomena in a hot plasma to be accurately modelled.In addition this approximation removes the non-local behaviour. In this paper $2 \mathrm{D}$ scattering off a plasma within a stationary magnetic field is studied. The excitation used throughout the paper is a TE polarized plane wave. In principle any fixed incoming field can be used. Intermediary results will be shown and suggestions for further research will be discussed.

\section{METHOD OF MOMENTS SOLUTION}

\section{Integral equation and discretization}

The plasma region is discretized with a uniform rectangular mesh. In each square the medium parameters are allowed to vary continuously. With every edge of the mesh an edge basis function will be associated to discretize the integral problem.

The wave problem in the cold plasma approximation can be solved solely using Maxwell's equations in sinus regime. Using the volume equivalence principle it is 
possible to derive a volume contrast integral equation (VCIE). Maxwell's equations can be rewritten for vacuum with an additional contrast source accounting for the plasma response. The next step is to write down the Helmholtz equation and to use the theory of Green's functions to obtain the VCIE. When the mixed potential expression for the Green dyadic is employed, the following equation is obtained:

$$
\begin{aligned}
\mathbf{e}_{\mathbf{t}}(\rho)= & \mathbf{e}_{\mathbf{t}}^{\text {in }}(\rho)+k_{0}^{2} \int_{S} G\left(\rho \mid \rho^{\prime}\right) \Delta \overline{\bar{\varepsilon}}_{t t}\left(\rho^{\prime}\right) \cdot \mathbf{e}_{t}\left(\rho^{\prime}\right) d S^{\prime} \\
& +\nabla_{t} \int_{S} G\left(\rho \mid \rho^{\prime}\right) \nabla_{t}^{\prime} \cdot\left(\Delta \overline{\bar{\varepsilon}}_{t t}\left(\rho^{\prime}\right) \cdot \mathbf{e}_{\mathbf{t}}\left(\rho^{\prime}\right)\right) d S^{\prime}
\end{aligned}
$$

Where $\Delta \overline{\bar{\varepsilon}}=\overline{\bar{\varepsilon}}-\overline{\bar{I}}$. The cold plasma dielectric tensor $\overline{\bar{\varepsilon}}$ is given by:

$$
\overline{\bar{\varepsilon}}_{t t}=\left(\begin{array}{cc}
\varepsilon_{t} & -\varepsilon_{c} \\
\varepsilon_{c} & \varepsilon_{t}
\end{array}\right)
$$

There are multiple ways of discretizing this integral equation. The possibility to choose $\Delta \overline{\bar{\varepsilon}}_{t t} \cdot \mathbf{e}_{\mathbf{t}}$ as the unknown field and to expand it in a divergence conforming basis is very tempting. Using divergence conforming RWG (Rao-Wilton-Glisson) basis functions would allow the divergence operator to be well defined. It was discovered though that this choice of basis doesn't allow for accurate results. The problem was identified to be caused by the basis functions not permitting the boundary conditions on the edge of the plasma-vacuum interface to be fulfilled. For non-continuous $\varepsilon$ profiles it can be shown that the boundary condition for the electric induction cannot be satisfied.

In this paper a different approach was opted for: the electric field is discretized using curl conforming RWG basis functions. However by doing so a new problem is introduced. After multiplication with the contrast tensor $\Delta \overline{\bar{\varepsilon}}_{t t}$ the divergence operator in the integral equation is ill-defined. To overcome this problem a completeness relation using a Gramian matrix is introduced (non-orthogonal base). After discretisation a set of linear equations remains.

$$
\begin{aligned}
\sum_{n=0}^{N-1} a_{n}\left[G_{d c}\right]_{m n}+\sum_{n, p, q=0}^{N-1} a_{n}<\mathbf{d}_{\mathbf{m}}|\mathscr{G}| \mathbf{d}_{\mathbf{p}}> & {\left[G_{c d}^{-1}\right]_{p q}<\mathbf{c}_{\mathbf{q}}|\Delta \overline{\bar{\varepsilon}}| \mathbf{c}_{\mathbf{n}}>} \\
& =<\mathbf{d}_{\mathbf{m}} \mid \mathbf{e}_{\mathbf{t}}^{\mathbf{i n}}>
\end{aligned}
$$

Hereby $\mid \mathbf{c}_{\mathbf{n}}>$ is identified as a curl conforming and $\mid \mathbf{d}_{\mathbf{m}}>$ as a divergence conforming basis function. After discretization this system can be written in a compact form as $Z \cdot X=B$.

Classicaly this system would be inverted using for example LU decomposition. The complexity of this process is $\mathscr{O}\left(N^{3}\right)$ with $\mathrm{N}$ being the number of unknowns used to discretize the system. Often there can be chosen for an iterative approach. Using Krylov Subspace methods allows for fast convergence of the iterative procedure. The convergence speed depends on the condition number of the system matrix and the clustering of the eigenvalues. If this is not optimal often a preconditioner can be added to the system to change the spread of the eigenvalues.

For large problems it is no longer possible to store the system matrix. Due to the 
translation symmetry of the background medium the integral operator $\mathscr{G}$ has a convolution structure. This implies that the matrix is Toeplitz. This means only the different interactions need to be stored, which has the consequence that memory complexity becomes: $\mathscr{O}(N)$. The system matrix is factorized into a Toeplitz matrix, the inverse of a Gramian and a matrix containing the dielectric contrast. It is not possible to store the inverse Gramian because this would destroy the favorable memory complexity. Therefore the inversion needs to be done iteratively in each loop. A careful choice of the basis and test set will assure the Gramian matrix to be well conditioned. In practice this means this sub-invertion in less than 5 iterations. For that reason the overall complexity of $\mathscr{O}(N \log N)$ is not altered.

\section{Accuracy}

The conforming basis set can be shown to fulfill the Babuska-Brezzi conditions [1] for accurate numerical respresentation of the wave solution. This implies that the upper boundary on the numerical error equals the interpolation error and can be made infinitesimally small by mesh refinement.

In simulating a reference system and comparing it with an analytical solution we see rapid convergence to the analytical result. The measurement points are located on a circle being in the far field of the cylindrical scatterer.

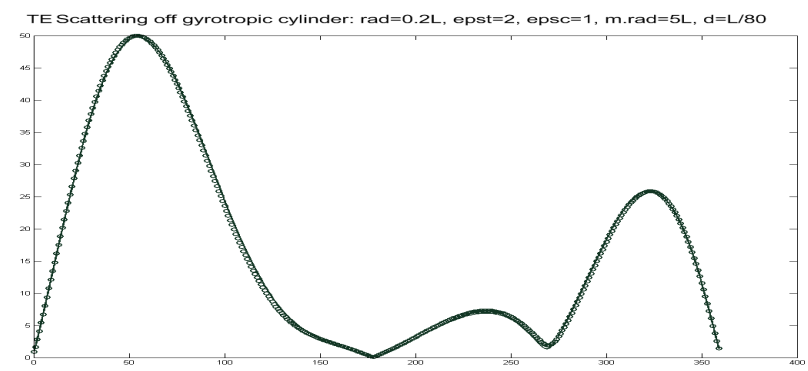

Figure 1. Comparison of the numerically obtained scattered far field for a gyrotropic cylinder with the analytic solution. The $\mathrm{x}$-axis shows the poloidal angle, the $\mathrm{y}$-axis the modulus of the field in $\mathrm{V} / \mathrm{m}$

\section{Computational speed}

The speed of the solver is determined by the product of the time for a matrix-vector product and the number of iterations. In this graph we will investigate if the predicted complexity of the matrix-vector product is obtained. It is experimentally confirmed that the theoretical $\mathscr{O}(N \log N)$ time complexity is achieved.

Next the speed of convergence is investigated as a function of the plasma dielectric parameters. It is observed that the number of iterations rises too fast to have a satisfying overall complexity. If the problem is well-conditioned the relation should be sublinear. 


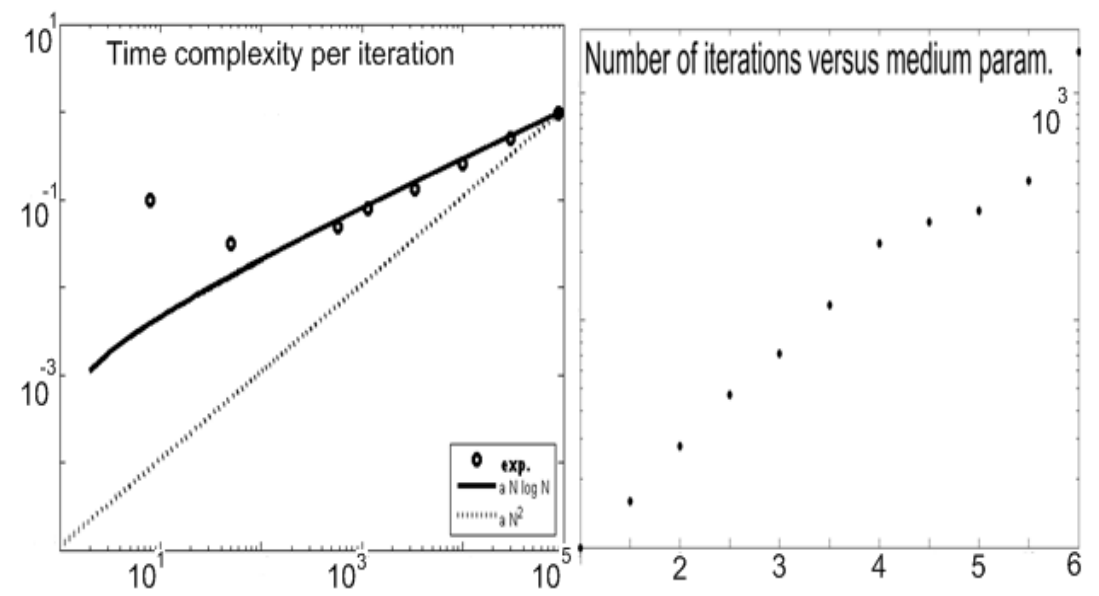

Figure 2. Left: Time complexity of matrix-vector product in function of number of unknowns N (loglog plot), Right: The number of iterations in function of the medium parameters for a fixed size cylinder. On the $\mathrm{X}$-axis is $\sqrt{\varepsilon_{t}^{2}+\varepsilon_{c}^{2}}$ (log-plot)

Volume integral equations tend to have a lot of resonances which may cause this behaviour.

\section{FUTURE RESEARCH}

At this moment simulations are going on to include the reactor vessel and low density regions. Other research will focus on developing a preconditioner to ameliorate the convergence of the iterative solver. To get rid of resonances it would be interesting to work with the combined volume integral equation, as this might solve the problem of the resonances which could be responsible for the ill-posedness of the problem. The comparison with finite element methods is going to be made to assure that this VCIE method shows competetive with FEM codes.

\section{REFERENCES}

1. I. Babuska, and K. A. Aziz, Lectures on mathematical foundations of finite element method, Univ. of Maryland, Washington DC, 1972.

\section{ACKNOWLEDGMENTS}

This research wouldn't exist without the continuous efforts of the other authors of this article. I would like to direct my special thanks towards Professor Olyslager who was my supervisor for over one year. Her ideas gave birth to this research project. It is very regrettable that she is not present to see her ideas being taken in to practice. To alot of people she will always be an inspiration. 